\title{
Optical fiber poling by induction: Analysis by 2D numerical modeling
}

\author{
F. De LuCIA, ${ }^{1 \S}$ D. Huang, ${ }^{2 \S}$ C. Corbari, ${ }^{1}$ N. Healy, ${ }^{3}$ P.J.A. SAZIO ${ }^{1, *}$ \\ ${ }^{1}$ Optoelectronics Research Centre, University of Southampton, SO17 1BJ, UK \\ ${ }^{2}$ Institute of High Performance Computing, A*STAR, 1 Fusionopolis Way, 138632, Singapore \\ ${ }^{3}$ Emerging Technology and Materials Group, Newcastle University, Merz Court, Newcastle, NE1 7RU, UK \\ $\S$ These authors contributed equally to this work \\ *Corresponding author: pjas@soton.ac.uk
}

Received XX Month XXXX; revised XX Month, XXXX; accepted XX Month XXXX; posted XX Month XXXX (Doc. ID XXXXX); published XX Month XXXX

Since their first demonstration some 25 years ago, thermally poled silica fibers have been used to realize device functions such as electro-optic modulation, switching, polarization entangled photons and optical frequency conversion with a number of advantages over bulk free-space components. We have recently developed an innovative induction poling technique that could allow for the development of complex microstructured fiber geometries for highly efficient $\chi^{(2)}$ based device applications. In order to systematically implement these more advanced poled fiber designs, we report here the development of comprehensive numerical models of the induction poling mechanism itself via 2D simulations of ion migration and space-charge region formation using finite element analysis. (C) 2016 Optical Society of America

OCIS codes: (190.4370) Nonlinear optics, fibers; (230.4320) Nonlinear optical devices; (190.2620) Harmonic generation and mixing; (230.1150) All-optical devices; (000.4430) Numerical approximation and analysis.

The development of thermal poling, a technique to generate effective second order nonlinearities in silica optical fibers [1], has found widespread applications in parametric frequency conversion [2], electro-optic modulation, switching [3] and polarization-entangled photon pair generation [4]. During thermal poling, the optical fiber is heated in order to increase the mobility of the impurity charge carriers (typically $\mathrm{Na}^{+}, \mathrm{Li}^{+}, \mathrm{K}^{+}$), while a high voltage is applied for a certain time between two electrodes embedded into the fiber [5]. The static electric field due to the application of the high voltage causes the impurity charges to drift from regions at high potential towards regions at lower potential creating a space charge region located near the anode. When the sample is cooled down whilst the voltage is still applied, an electric field is frozen into the depleted region and an effective nonlinear susceptibility $\chi_{\text {eff }}^{(2)}$ is induced into the sample due to a process of third order nonlinear optical rectification. The early issues mainly related to the high risk of breakdown between the two electrodes (typically separated by a few tens of microns) were addressed by Margulis et al. [6], who demonstrated that it is possible to induce a value of $\chi_{\text {eff }}^{(2)}$ higher than the one obtained in the conventional case [5] by means of a poling configuration in which the two embedded electrodes are both connected to the same positive potential of the anode. The method for "charging" optical fibers has been recently further developed by De Lucia et al. [7], who discovered that it is possible to create a space charge region using electrostatic induction between an external inductor and the floating electrodes embedded inside a fused silica twin-hole fiber. As this novel technique avoids any physical contact to the internally embedded electrodes, it automatically lifts a number of restrictions on the use of microstructured optical fibers for poling where the multiple contacting of individual electrodes becomes a prohibitive challenge. Thus the induction poling technique, together with the use of embedded liquid electrodes such as Gallium [7], could, for example, allow for the poling of complex photonic crystal fibers (PCF), with the aim of realizing devices that fully exploit the inherently desirable PCF properties of strong optical mode confinement, dispersion, effective group index engineering, etc for highly efficient nonlinear functionality.

In order to systematically implement these advanced device concepts, it is first necessary to develop comprehensive numerical models of the induction poling mechanism itself. To this end, we report here the development of 2D simulations of induced spacecharge region formation using $\mathrm{COMSOL}^{\circledR}$ finite element analysis. This builds on current numerical models by Camara et al. [8] who developed a two-dimensional analysis that accurately describes the specific geometry of poled fibers with internal electrodes, thus highlighting the role of various cations and the time evolution of second-order nonlinear profile within the fiber geometry. However, our recent experimental demonstration of electrostatic induction poling suggests that substantial modifications to the boundary conditions of these current 2D models are required in order to fully elucidate this novel poling mechanism. In the first instance, this arises due to the inherent assumption [8] that the 
outer surface of the fiber is always at ground potential. While this is a reasonable assumption when considering the experimental setup described by Margulis et al. [6], an external field applied by an inductor to floating electrodes inside fibers would, by definition, be fully shielded by this grounded surface, thus completely inhibiting the induction poling process. Furthermore, the model of Camara et al. [8] concludes that field-dependent $\mathrm{H}_{3} \mathrm{O}^{+}$ion injection, which only becomes significant for longer poling times, does not differ significantly from constant rate injection conditions, given that the electric field at the anode-glass boundary reaches a value of $\sim 10^{9} \mathrm{~V} / \mathrm{m}$ over a short time period and then remains constant. However, in the induction poling geometry, the variable floating potentials inherent in this process require a field dependent charge injection scheme. Finally, it is also necessary to modify the fielddependency to take into account ion recombination at the cladding-air interface. We show that mapping the salient features of the induction poling experimental geometry can allow us draw a more appropriate set of boundary conditions and the modified 2D model can then be used to describe the dynamics of the secondorder nonlinearity created inside the fiber when it is both close to and far away from the external inductor (as shown in Fig. 1).

As described in $[8,9]$, Sodium $\left(\mathrm{Na}^{+}\right)$is the cation of highest mobility in silica and its displacement results in a cation-depleted region of high electrical resistivity near the floating electrodes embedded inside a fused silica twin-hole fiber waveguide. The negatively charged, non-bridging oxygen sites left behind (uncompensated negative charges) have very low mobility at 300 ${ }^{\circ} \mathrm{C}$ and it is this charge separation that gives rise to the recorded field. The ions reaching the electrode-cladding surface or the aircladding surface are assumed to recombine on these surfaces, rather than accumulate. If poling continues for long enough, the width of the depletion region increases but other slower cations are also driven into the glass (e.g. $\mathrm{H}_{3} \mathrm{O}^{+}$), which begin to neutralize the uncompensated negative charges. When both migration and diffusion are taken into account, the local equation of continuity and Poisson's equation leads to the following partial differential equation solved in $x, y$ and $t$ :

$$
\frac{\partial c_{i}}{\partial t}+\nabla \cdot\left(-D_{i} \nabla c_{i}-z_{i} \mu_{i} F c_{i} \nabla V\right)=R_{i}
$$

where the index i represents one of the positive ionic species $(i=$ 1 for sodium ions and $\mathrm{i}=2$ for the injected charges) and the first term in brackets accounts for diffusion and the other term corresponds to drift in the electric field. In Eq. (1), c is the concentration, $\mathrm{D}$ the diffusivity, $\mathrm{z}$ the charge, $\mu$ the ion mobility, $\mathrm{F}$ is Faraday's constant, $\mathrm{V}$ the electric potential and $\mathrm{R}$ the consumption or production rate. The electric field and potential distribution as a function of poling duration are derived from Maxwell's equations in the electrostatic regime, from which it is possible to deduce the second-order nonlinearity spatial distribution within the fiber.

For the implementation of the COMSOL ${ }^{\circledR}$ model, a twin-hole fiber with dimensions identical to our experimentally relevant samples described in ref [7] (i.e. OD $=125 \mu \mathrm{m}$, core diameter 4.3 $\mu \mathrm{m}, \mathrm{NA}=0.17$ ) is enclosed by a virtual "air box" as shown in Fig. 2 . An inductor placed adjacent to the fiber provides the external electric field that charges up the floating electrodes inside (see Fig. 1). The uniformly distributed $\mathrm{Na}^{+}$ions are assumed to have an initial concentration of $1 \mathrm{ppm}$ at $\mathrm{t}=0 \mathrm{~s}$. It is also assumed that there is up to $1 \mathrm{ppm}$ of $\mathrm{H}_{3} \mathrm{O}^{+}$ions injectable at the holes, with none present within the fiber at $t=0 \mathrm{~s}$. In order to achieve initial charge neutrality, negatively charged, non-bridging oxygen sites (i.e. NBO-
) with extremely low mobility are uniformly distributed inside the fiber with a concentration of $1 \mathrm{ppm}$ at $\mathrm{t}=0 \mathrm{~s}$.

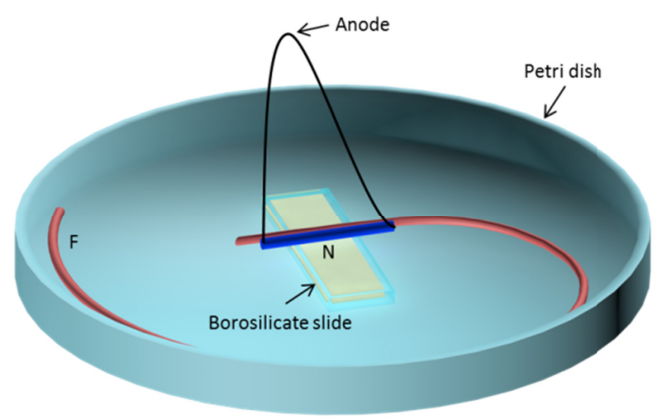

Fig. 1. Schematic of the experimental setup for induction poling of optical fibers. The inductor is colored blue (equipped with two electrodes connected to the same anodic potential) with the sample colored red (with two embedded floating electrodes) to be poled by induction. Both are adhered to a glass slide and held in close proximity. $N$ identifies a region of the sample near the inductor, $F$ a section of the same sample located far away from the inductor. The entire Petri dish is heated with a (electrically earthed) hotplate at $300^{\circ} \mathrm{C}$.

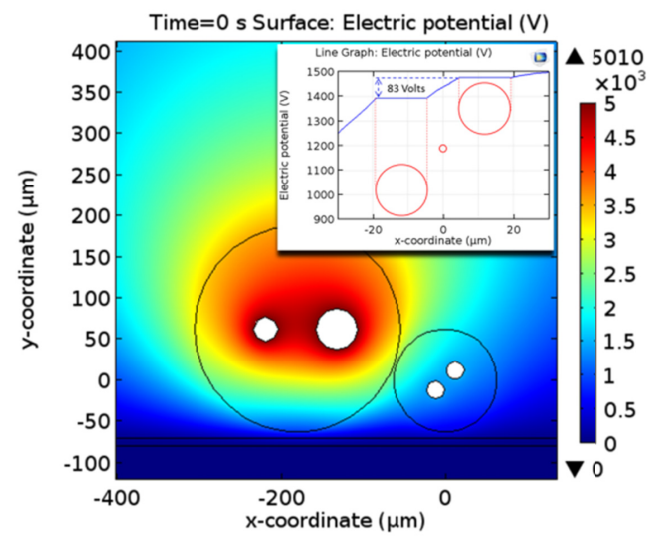

Fig. 2. 2D representation of the initial $(t=0 \mathrm{~s})$ electric potential distribution developed within the electrostatic induction poling mechanism. The setup consists of the external inductor on the LHS, comprised of twin electrodes at $+5 \mathrm{kV}$ running through a $6 \mathrm{~cm}$ long silica fiber dielectric sheath as shown in Fig. 1 to prevent unwanted electrical breakdown to a grounded heater underneath. The sample being poled on the RHS is described in ref [7] and is rotated at an arbitrary angle with respect to the inductor in order to replicate realistic experimental conditions. The inset shows the electric potential values recorded along a line scan through the geometric centers of the sample, exhibiting a potential difference of around $83 \mathrm{~V}$.

The holes are also assumed to be entirely filled with metal electrodes and present an equipotential. Only the outer boundaries of the "air box" (a cube with side $10 \mathrm{~cm}$ ) and the ground plane (presented by the metallic heater) located $10 \mu \mathrm{m}$ underneath the inductor and fiber sample in Fig. 2 are assumed to be electrically earthed. Furthermore, $\mathrm{H}_{3} \mathrm{O}^{+}$ions can be injected through the electrode-cladding surface when located at electric potentials higher than the surrounding cladding. An adjustable parameter $\sigma_{2}$ 
(whose value was chosen to be the same as the well established thermal poling model reported in [9]) is used to describe the charge injection into the fiber. We also need to consider the special case where the electric field is less than zero. In this case, $\mathrm{H}_{3} \mathrm{O}^{+}$ions in the nearby cladding (either previously injected or diffused from other parts of the fiber) will have a negative injection rate, thus implying an outflow of $\mathrm{H}_{3} \mathrm{O}^{+}$ions. However, if there are not any $\mathrm{H}_{3} \mathrm{O}^{+}$ions (i.e. $\mathrm{c}\left(\mathrm{H}_{3} \mathrm{O}^{+}\right)=0$ ) at the electrode-cladding boundary, the injection rate will be zero even with an electric field less than zero. Therefore, the variation of the injected $\mathrm{H}_{3} \mathrm{O}^{+}$ions density per unit of time at the electrode-cladding surface can be written as [9]:

$$
\begin{aligned}
& \left.\left(\frac{\partial c_{2}}{\partial t}\right)\right|_{\text {surface }}=\sigma_{2} E, E \geq 0 \text { or } E<0 \text { and } c_{2}>0 \\
& \left.\left(\frac{\partial c_{2}}{\partial t}\right)\right|_{\text {surface }}=0, \text { otherwise }
\end{aligned}
$$

where $E$ is the electric field magnitude at the electrode surface.
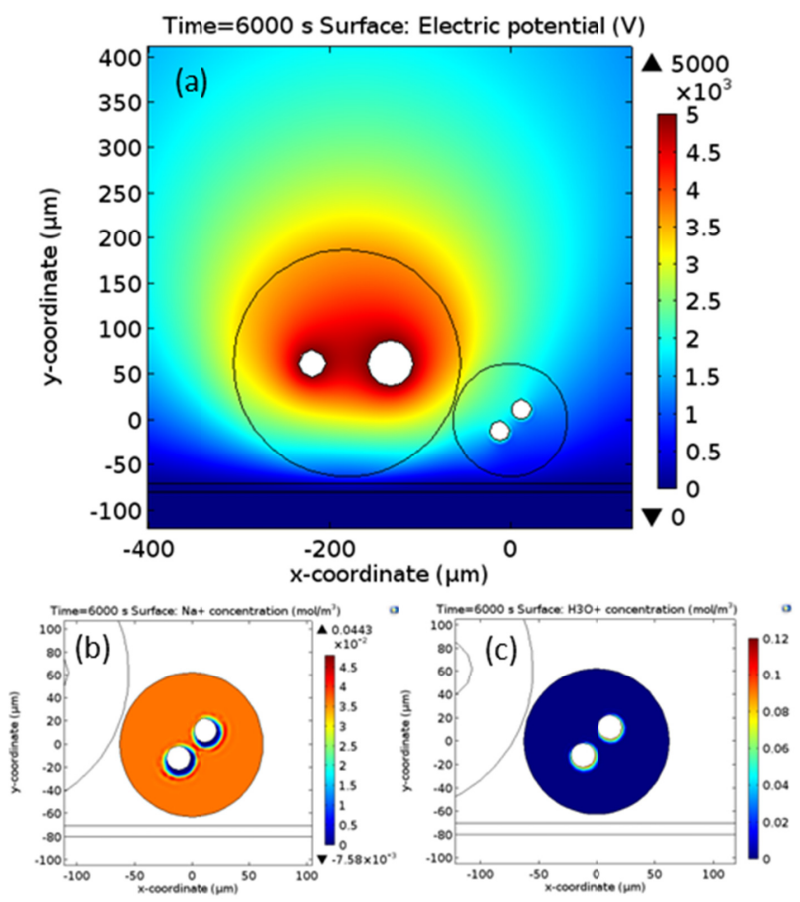

Fig. 3. Distribution of (a) electric potential, (b) $\mathrm{Na}^{+}$and (c) $\mathrm{H}_{3} \mathrm{O}^{+}$mobile cations after 100 mins of poling near the inductor (position $N$ in Fig. 1).

The avalanche-like, positive feedback depletion region formation as described by Margulis et al. [6] in "cathode-free" fibers is also considered to operate in an enhanced form within the electrostatic induction poling mechanism [7]. However, within this configuration, it is important to note that there are two distinct and separate spatial regimes. Firstly, we can examine the evolution of the depletion region formation when the fiber sample is immediately adjacent to the external inductor as shown in Fig. 3, where the electric field from the inductor penetrates inside the fiber sample as it lacks a grounded outer surface in contrast to existing thermal poling models. As can be seen, after 100mins the electric potential distribution developed inside the fiber sample and subsequent cation migration leads to an asymmetry in comparing both the size and shape of the depletion regions formed around the internal floating electrodes. This disparity reflects the inhomogeneity of the electric field distribution close to the inductor and the strong influence of the nearby ground plane defined by the heater (and the relatively low series resistance of the borosilicate glass slide and Petri dish at elevated temperatures in Fig. 1). The summation of sodium and hydronium ion depletion regions, where $\mathrm{H}_{3} \mathrm{O}^{+}$ions occupy the negatively charged sites left by migrating $\mathrm{Na}^{+}$ions, results in a circular negative net charge distribution layer [8] surrounding the floating electrodes that will be asymmetric as detailed above.
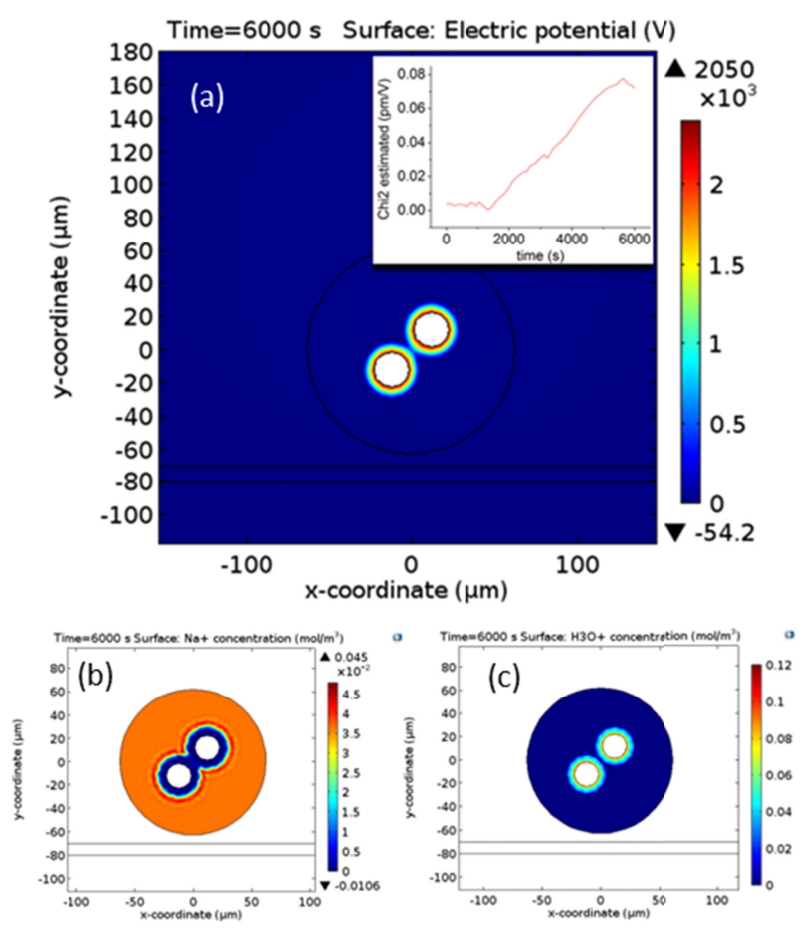

Fig. 4. Distribution of (a) electric potential, (b) $\mathrm{Na}^{+}$and (c) $\mathrm{H}_{3} \mathrm{O}^{+}$mobile cations after 100 mins of poling far from the inductor (position $F$ in Fig. 1). The inset of (a) shows the evolution of the second order nonlinearity as derived from the magnitude of the electric field at the center of the fiber where the $4.3 \mu \mathrm{m}$ diameter, NA $=0.17$ Germania doped core is located, as described in ref [7].

As well as this specific behavior close to the $(6 \mathrm{~cm}$ long) inductor, it is also possible to model the poling mechanism some distance from this region as typically the sample being poled is many times longer than the inductor itself (see ref [7] and Fig. 1). In this spatially separated scenario, it is valid to assume that the electric field due to the inductor no longer has any direct influence on the cation migration within the remainder of the sample. Nevertheless, due to the fact that during induction poling, there is very little if any, current flow through the fiber and therefore virtually no voltage drop due to metallic series resistance, the internal floating electrodes will present a well characterized equipotential surface throughout the entire sample. This means that any electric potential picked up by the floating electrodes close to the inductor will be effectively transferred to any remote location throughout the fiber. In addition, this electric potential will constantly evolve due to the temporal evolution of the induction poling dynamics. In other words, the model used to describe the induction poling mechanism far from the inductor can be considered as similar to 
that developed by Camara et al. [8]. Important differences remain however, such as the position of the ground potential, which rather than being the outer surface of the fiber sample, is defined instead as the outer boundaries of a virtual "air box" as well the electrically earthed heater (combined with the low borosilicate glass series resistance) as shown in Fig. 4. Furthermore, the electric potential of each internal electrode is not a value fixed at $t=0$ as in ref [8], but rather varies continuously as defined by the electric potential distribution close to the inductor.

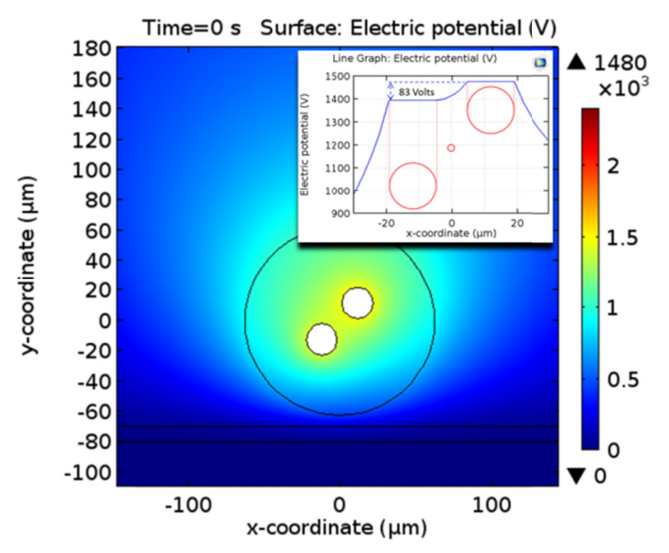

Fig. 5. Distribution of the initial $(\mathrm{t}=0 \mathrm{~s})$ electric potential far from the inductor. The inset shows the electric potential values recorded along a line scan through the geometric centers of the sample, exhibiting a potential difference value of around $83 \mathrm{~V}$, just as in the inset of Fig. 2.

It is interesting to note how the model both close to (Fig. 2) and far from (Fig. 5) the inductor exhibits a strong inhomogeneity of the electric field distribution around the floating electrodes as well as a strong influence due to the earthed heater. This suggests that an enhanced "avalanche-like" positive feedback $[6,7]$ drives the formation of the depletion region. Furthermore it is worth noting that, even if the electric equipotential surfaces of the floating electrodes near and far from the inductor evolve in time in the same way, the two depletion regions develop differently in the two different locations. This is due to the presence of the external electric field of the inductor, which modifies the total electric field distribution around each electrode compared to the situation far from the inductor. A different distribution of the electric field in the space around the electrodes will determine a different evolution of the depletion region in these positions. As shown in Fig. 6, our simulations also reveal that the relative azimuth rotation angle of the sample (with respect to the inductor fiber and underlying heater) as well as the position of the core with respect to the electrodes can further enhance the internal potential difference demonstrating that induction poling can be engineered and optimized with many different parameters. In summary, we have presented the results of 2D simulations [10], developed using COMSOL Multiphysics ${ }^{\circledR}$ finite element analysis of space-charge region formation generated via electrostatic induction poling. Substantial modifications to the boundary conditions of current numerical models were required in order to fully elucidate this novel poling mechanism. This modified 2D model can be used to understand the dynamics of the second order nonlinearity created inside the fiber sample due to depletion region formation when it is both close to and far from the inductor. Our numerical analysis presents us with a powerful general method to develop models of induction poling within any complex geometry, such as for example, microstructured optical fibers that potentially allow up to four orders of magnitude improvements in second harmonic generation efficiency relative to conventionally poled step-index optical fibers [11].

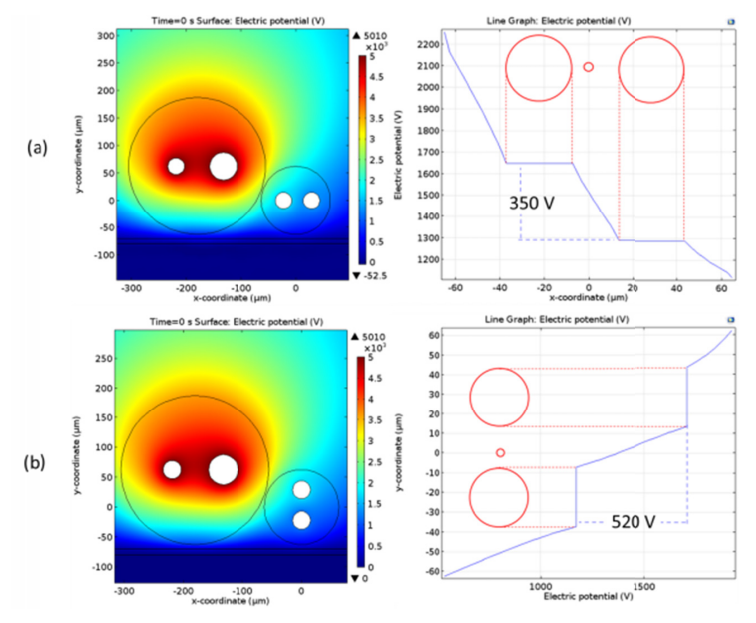

Fig. 6. (Left) Simulated initial 2D electric potential distribution before charged ion migration begins to generate the depletion region. The external inductor and adjacent sample fiber are shown with zero relative azimuthal rotation. (Right) A line scan through the geometric centers of the sample reveals large potential variations $(350 \mathrm{~V})$ that drives impurity ion migration during electrostatic induction poling. (b) (Left) 2D electric potential distribution for a 90 degree relative azimuthal rotation of the sample. (Right) A line scan through the geometric centers of this sample orientation exhibits an even larger $(520 \mathrm{~V})$ potential difference between the internal electrodes compared with the sample in (a), resulting in faster depletion region dynamics.

\section{Funding. EPSRC EP/I035307/1}

\section{References}

1. R. A. Myers, N. Mukherjee, and S. R. J. Brueck, Opt. Lett. 16, 1732 (1991).

2. A. Canagasabey, C. Corbari, A. V. Gladyshev, F. Liegeois, S. Guillemet, Y. Hernandez, M. V. Yashkov, A. Kosolapov, E. M. Dianov, M. Ibsen, and P. G. Kazansky, Opt. Lett. 34, 2483 (2009).

3. M. Malmstrom, O. Tarasenko and W. Margulis, Opt. Exp. 20, 9465 (2012).

4. E. Y. Zhu, Z. Tang, L. Qian, L. G. Helt, M. Liscidini, J. E. Sipe, C. Corbari, A. Canagasabey, M. Ibsen, and P. G. Kazansky, Opt. Lett. 38, 4397 (2013).

5. D. Wong, W. Xu, S. Fleming, M. Janos, and K-M Lo, Opt. Fib. Tech. 5, 235 (1999).

6. W. Margulis, O. Tarasenko and N. Myrén, Opt. Exp. 17, 15534 (2009).

7. F. De Lucia, D. Huang, C. Corbari, N. Healy and P. Sazio, Opt. Lett. 39, 6513 (2014).

8. A. Camara, O. Tarasenko, and W. Margulis, Opt. Exp. 22, 17700 (2014).

9. A. Kudlinski, Y. Quiquempois, and G. Martinelli, Opt. Exp. 13, 8015 (2005).

10. F. De Lucia, D. Huang, C. Corbari, N. Healy and P. J. A. Sazio, "Optical fiber poling by induction: Analysis by 2D numerical modelling [dataset]" (University of Southampton, 2016) http://dx.doi.org/10.5258/SOTON/386031

11. T. M. Monro, V. Pruneri, N. G. R. Broderick, D. Faccio, P. G. Kazansky and D. J. Richardson, IEEE Photon. Tech. Lett. 13, 981 (2001). 


\section{References}

1. R. A. Myers, N. Mukherjee, and S. R. J. Brueck. "Large second-order nonlinearity in poled fused silica." Opt. Lett., 16, no. 22 (1991): 1732 1734.

2. A. Canagasabey, C. Corbari, A. V. Gladyshev, F. Liegeois, S. Guillemet, Y. Hernandez, M. V. Yashkov, A. Kosolapov, E. M. Dianov, M. Ibsen, and P. G. Kazansky. "High-average-power second-harmonic generation from periodically poled silica fibers." Opt. Lett. 34, no. 16 (2009): 2483-2485.

3. M. Malmstrom, O. Tarasenko and W. Margulis. "Pulse selection at 1 MHz with electrooptic fiber switch." Opt. Exp. 20, no. 9 (2012): 94659470.

4. E. Y. Zhu, Z. Tang, L. Qian, L. G. Helt, M. Liscidini, J. E. Sipe, C. Corbari, A. Canagasabey, M. Ibsen, and P. G. Kazansky. "Poled-fiber source of broadband polarization-entangled photon pairs." Opt. Lett. 38, no. 21 (2013): 4397-4399.

5. D. Wong, W. Xu, S. Fleming, M. Janos, and K-M Lo. "Frozen-in electrical field in thermally poled fibers", Opt. Fib. Tech. 5 (1999): 235 - 241.

6. W. Margulis, O. Tarasenko and N. Myrén. "Who needs a cathode? Creating a second-order nonlinearity by charging glass fiber with two anodes." Opt. Exp. 17, no. 18 (2009): $15534-15540$.

7. F. De Lucia, D. Huang, C. Corbari, N. Healy and P. Sazio. "Optical fiber poling by induction." Opt. Lett. 39, no. 22 (2014): 6513-6516.

8. A. Camara, O. Tarasenko, and W. Margulis. "Study of thermally poled fibers with a two-dimensional model." Opt. Exp. 22, no. 15 (2014): 17700-17715.

9. A. Kudlinski, Y. Quiquempois, and G. Martinelli. "Modeling of the $\chi^{(2)}$ susceptibility time-evolution in thermally poled fused silica. "Opt. Exp. 13, no. 20 (2005): 8015-8024.

10. F. De Lucia, D. Huang, C. Corbari, N. Healy and P. J. A. Sazio, "Optical fiber poling by induction: Analysis by 2D numerical modelling [dataset]" (University of Southampton, 2016) http://dx.doi.org/10.5258/SOTON/386031

11. T. M. Monro, V. Pruneri, N. G. R. Broderick, D. Faccio, P. G. Kazansky and D. J. Richardson. "Broad-Band Second-Harmonic Generation in Holey Optical Fibers." IEEE Photon. Tech. Lett. 13, no. 9 (2001): 981 983. 